\title{
A Case of Lung Adenocarcinoma with Pulmonary Hypertrophic Osteoarthropathy Showing Pathological Complete Response to a Pembrolizumab-Containing Chemoimmunotherapy
}

\author{
Naoki Kataoka Shoji Oura Tomoyuki Yamaguchi Shinichiro Makimoto \\ Department of Surgery, Kishiwada Tokushukai Hospital, Kishiwada-city, Japan
}

Keywords

Immune checkpoint inhibitor - Non-small cell lung cancer - Pathological complete response . Pembrolizumab · Pulmonary hypertrophic osteoarthropathy

\begin{abstract}
A 65-year-old woman with prolonged cough and presumed pulmonary hypertrophic osteoarthropathy was referred to our hospital. Computed tomography showed 2 tumors larger than $3 \mathrm{~cm}$ in size and massive hilar lymph node enlargement in the right lung. Pathological examination of the transbronchial lung biopsy specimen showed atypical malignant cells, presumed adenocarcinoma, with $1 \%$ positivity of programmed cell death 1 ligand (PD-L1). Three courses of chemoimmunotherapy with pembrolizumab (400 mg q3w), carboplatin (AUC $5 \mathrm{mg}$ / $\mathrm{mL} \cdot \min \mathrm{q} 3 \mathrm{w})$, and pemetrexed $\left(500 \mathrm{mg} / \mathrm{m}^{2} \mathrm{q} 3 \mathrm{w}\right)$ were well tolerated and brought about a quasi-complete response both of the lung tumors and lymph nodes and complete symptom relief of the pulmonary hypertrophic osteoarthropathy, finally leading to the surgical intervention, that is, lobectomy and lymph node dissection. Postoperative pathological examination showed no viable cancer foci both in the lung tumors and lymph nodes. The patient recovered uneventfully. Physicians should note the combination chemoimmunotherapy including pembrolizumab, with curative intent, to optimally treat patients with locally advanced non-small cell lung cancer (NSCLC) even if the NSCLC bears a small amount of PD-L1.




\section{Case Reports in Oncology}

\begin{tabular}{l|l}
\hline Case Rep Oncol 2021;14:1380-1386 \\
\hline DOI: 10.1159/000512818 & $\begin{array}{l}\text { @ 2021 The Author(s). Published by S. Karger AG, Basel } \\
\text { www.karger.com/cro }\end{array}$ \\
\hline
\end{tabular}

Kataoka et al.: Lung Adenocarcinoma with Complete Response to a PembrolizumabContaining Chemoimmunotherapy

\section{Introduction}

Lung cancer is the leading cause of cancer-related mortality in men worldwide. Surgery has been the mainstay in the treatment of resectable non-small cell lung cancer (NSCLC) with curative intent. On the other hand, systemic therapy, especially chemotherapy, has played a major role in the treatment of inoperable NSCLCs. However, no effective systemic therapies, leading to a cure without local therapy, have been thus far established.

Immune tolerance through the interaction between programmed cell death 1 (PD-1) and programmed cell death 1 ligand (PD-L1) has become the important therapeutic target in the treatment of locally advanced or metastatic NSCLCs [1]. The advent of immune checkpoint inhibitors has dramatically changed the strategy and clinical outcome of inoperable NSCLCs.

We herein report a case of locally advanced NSCLC with pulmonary hypertrophic osteoarthropathy [2] showing a complete pathological response to a pembrolizumab-containing chemoimmunotherapy.
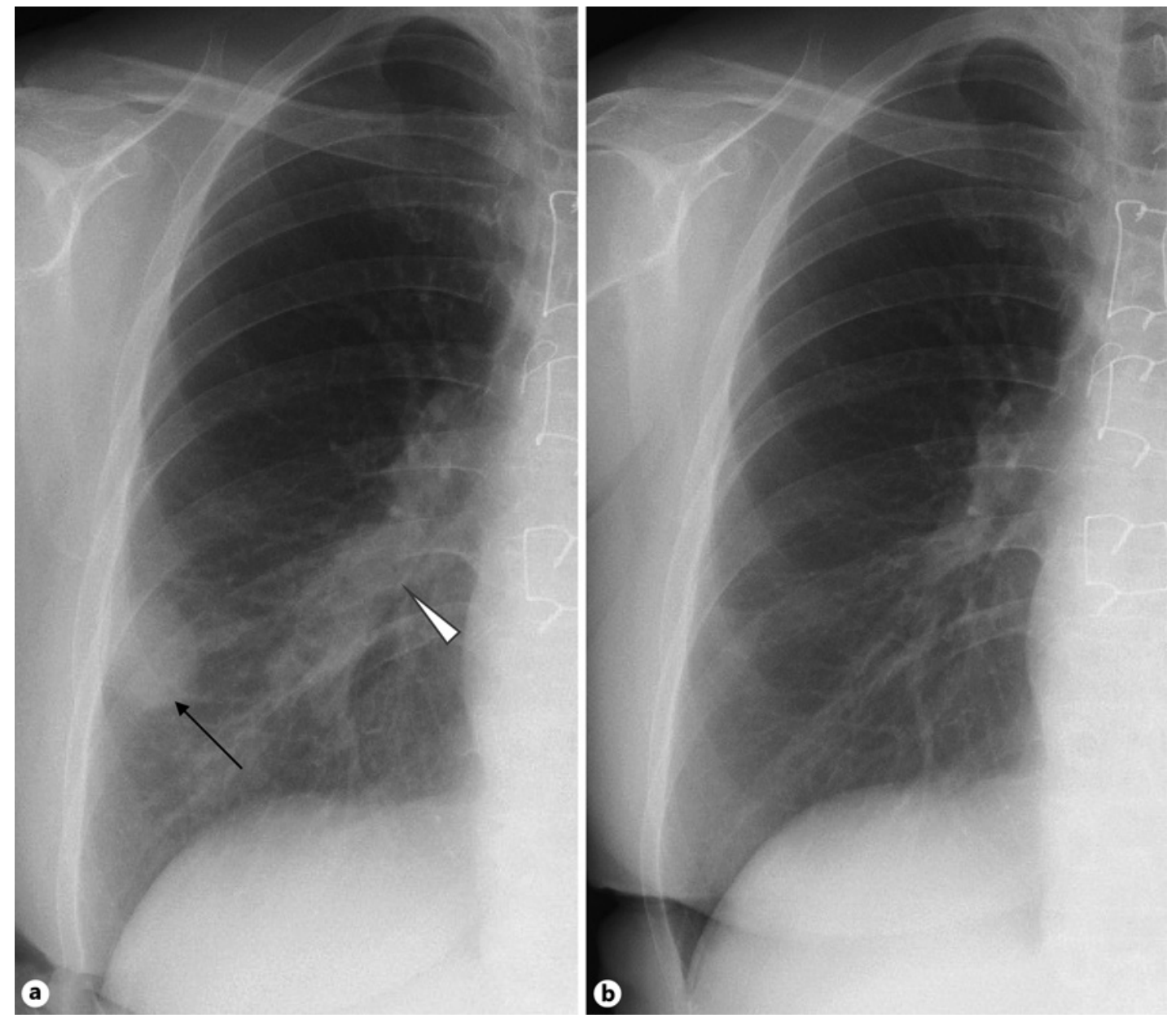

Fig. 1. Chest X-ray. a Chest X-ray before chemoimmunotherapy shows an oval shadow approximately $3 \mathrm{~cm}$ in size in the right lower lung field (arrow) and enlargement of the lung hilum (arrowhead). b Chest X-ray after chemoimmunotherapy showed complete disappearance of the tumor and shrinkage of the lung hilum. 

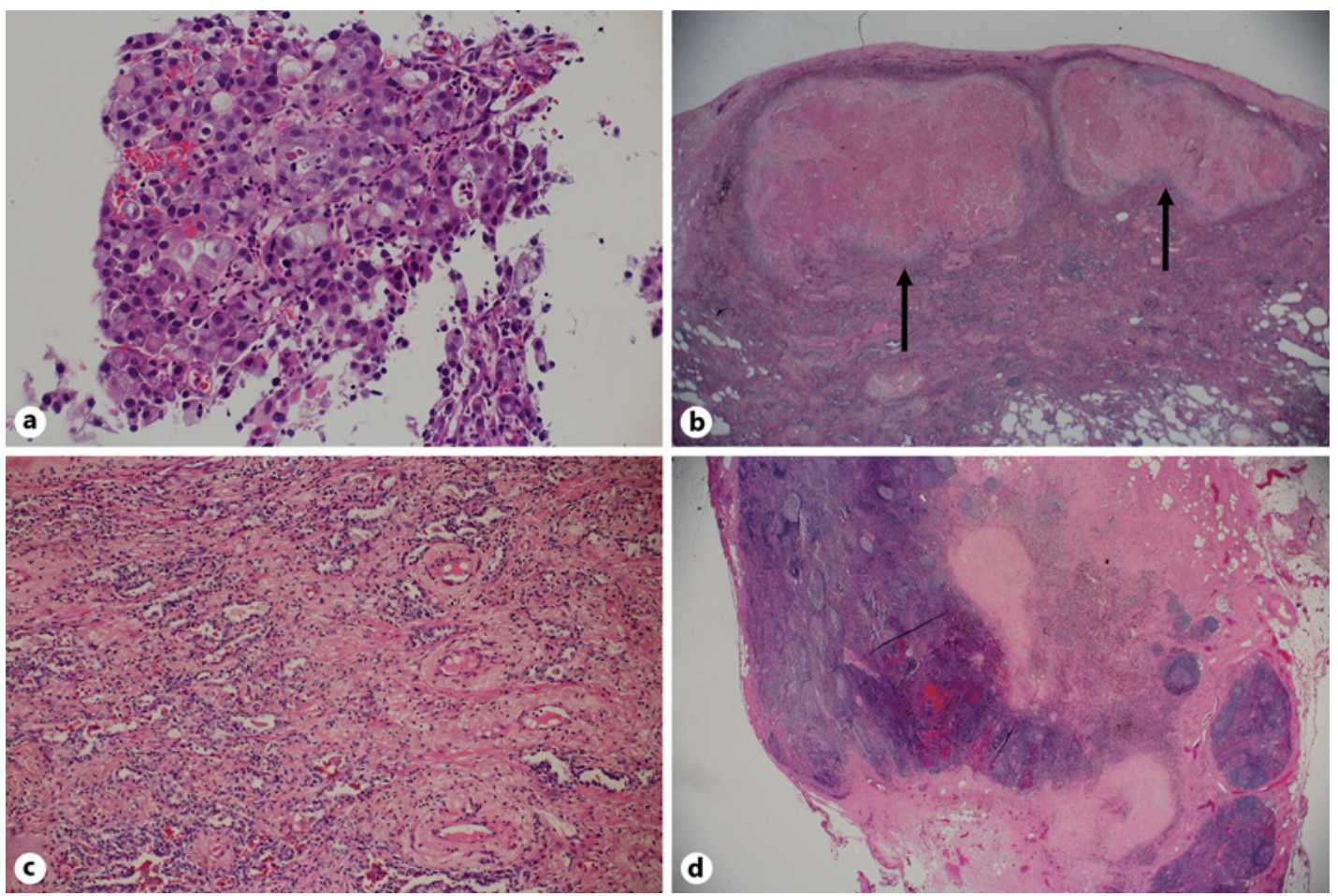

Fig. 2. Pathological examination. a Pathological examination of the transbronchial lung biopsy specimen showed the growth of large oval atypical cells in a sheet-like fashion. Some cells contain mucin in the cytoplasm. HE. $\times 200$. b Low magnification view of the resected specimen showed marked hyalinization (arrows) just beneath the pleura. HE. $\times 40$. c Higher magnification view of the resected specimen showed marked fibrosis and lymphocytic infiltrates with no viable cancer foci. HE. $\times 100$. d Low magnification view showed marked fibrosis and no viable cancer foci in the subcarinal lymph nodes dissected. HE. $\times 40$.

\section{Case Report}

A 65-year-old woman, an ex-smoker with a Brinkman index of 800, with a past history of breast cancer, urothelial cancer, and ischemic heart disease presented cough for several months. Plain chest X-P showed a mass in the right lung field and enlargement of the right lung hilum (Fig. 1). Computed tomography (CT) showed well-defined oval masses in the lung with apparent hilar lymph node enlargement. Laboratory testing showed no significant tumor marker elevations. Pathological examination of the transbronchial lung biopsy specimen showed large oval atypical cells with mucin formation in some cells. Immunostaining showed that the atypical cells (Fig. 2) were TTF-1, napsin A, CK5/6, CD56, synaptophysin, and chromogranin negative, Alucian blue positive, p40 and p63 focally positive, and PD-L1 weakly, that is, $1 \%$, positive. Molecular testing showed that no molecular mutations were observed in epidermal growth factor receptor exons 18-21. Positron emission tomography (PET) showed maximum standard uptake values of 8.0 and 14.1 in the 2 tumors located in the right lower lobe and those of 6.3 and 5.3 in the hilar and mediastinal lymph nodes, respectively, but no significant accumulation in the bone (Fig. 3). The patient with digital clubbing and cortical and periosteal thickening of the tibias complained of polyarthralgia after the onset of cough, leading to the diagnosis of pulmonary hypertrophic osteoarthropathy. The presence of 2 lung cancers in the right lower lobe suggested the T category in this case as at least T2, that is, more than $3 \mathrm{~cm}$ in size, or T3 with possible pulmonary metastasis in the same lobe. In addition, 



Fig. 3. PET/CT. a PET before chemoimmunotherapy showed 2 lung tumors (arrows) and hilar and mediastinal lymph nodes (arrowheads) with markedly increased avidity and no uptakes in the bone. b PET after chemoimmunotherapy showed no uptake in the lung tumors and lymph nodes. c PET/CT before chemoimmunotherapy showed increased avidity both in the lung tumor and the presumed hilar metastatic lymph node. d PET/CT after chemoimmunotherapy showed quasi-complete disappearance of the lung tumor and marked shrinkage of the hilar lymph node.

bulky presumed hilar lymph node metastasis could lead to inevitable pneumonectomy when primary surgery was applied to the patient. We, therefore, treated the patient with primary chemoimmunotherapy using pembrolizumab ( $400 \mathrm{mg} \mathrm{q} 3 \mathrm{w}$ ), carboplatin (AUC $5 \mathrm{mg} / \mathrm{mL} / \mathrm{min}$ $\mathrm{q} 3 \mathrm{w})$, and pemetrexed $\left(500 \mathrm{mg} / \mathrm{m}^{2} \mathrm{q} 3 \mathrm{w}\right)$. The patient showed prompt symptom relief, that is, disappearance of the cough and polyarthralgia with manageable side effects. Due to the quasi-complete remission of the lung cancers and the marked shrinkage of the presumed lymph node metastases (Fig. 3) with 3 courses of the chemoimmunotherapy, the patient underwent lower lobe lobectomy and hilar and mediastinal lymph node dissection. Patho- 
logical examination showed no viable cancer remnants in the lung tumors and lymph nodes dissected (Fig. 2). The postoperative course was uneventful and the patient was discharged on the 9th day after operation.

\section{Discussion}

Various therapies, mainly chemotherapy and molecularly targeted drugs such as bevacizumab [3], have been employed to improve survival of the patients with metastatic or locally advanced NSCLC, resulting in certain but nominal survival benefit with considerable therapyinduced uncomfortable side effects. Unlike breast cancers, pathological complete response has been regarded as exceptional in the treatment of locally advanced NSCLCs [4]. This case, however, showed a complete pathological response both in the lung cancers and presumed metastatic lymph nodes.

Single or dual immune checkpoint inhibitor therapy, much less toxic than conventional chemotherapies, has become a preferred option in the treatment of chemonaive inoperable NSCLC with high PD-L1 expression [5]. Various studies have showed that high PD-L1 expression correlated well with the favorite efficacy of the single or dual immune checkpoint inhibitor therapy against NSCLC on one hand. This case had only 1\% positivity of PD-L1 in the biopsy specimen but showed marked antitumor response clinically and pathologically on the other hand. A clinical trial, however, clearly showed that even though patients were limited to those with less than 1\% PD-L1 positive squamous cell carcinoma, adding pembrolizumab to conventional calboplatin and paclitaxel could lead to a significant survival benefit [6]. Immune checkpoint inhibitors, therefore, may be possible enhancers of the chemotherapy with unproven mechanisms. In fact, the regimen used in this case has received US Food and Drug Administration approval for the frontline metastatic NSCLC irrespective of PD-L1 expression.

Pulmonary hyperplastic osteoarthropathy is defined as the syndrome which mainly consisted of clubbed fingers, periostitis of the long tubular bones, and arthritis [2]. Patients with pulmonary hyperplastic osteoarthropathy, therefore, usually present a painful arthropathy. In this case, in addition to the amelioration of the cough with the tumor regression, pain as a symptom of paraneoplastic syndrome disappeared completely during the administration of pembrolizumab-containing chemoimmunotherapy. The presence of paraneoplastic syndrome, more often observed in small cell carcinoma than in NSCLC [7], generally implies that a subset of cancer cells, releasing some kind of symptom-causing cytokine(s), predominates in the tumor. Therefore, some anticancer therapy, when being effective for cancer clones causing paraneoplastic syndrome, could lead to marked antitumor effects, occasionally leading to a pathological complete response like this case. Therefore, patients with paraneoplastic syndrome due to NSCLC should be good candidates for multidisciplinary therapy with curative intent.

Unlike chemotherapy, immune checkpoint inhibitors generally develop unique adverse effects, so-called immune-related adverse effects, such as colitis, pneumonitis, hepatitis, neurotoxic effects, and myocarditis [8]. Fatal adverse effects were observed in approximately $0.4 \%$ of patients treated with an anti-PD-1/anti-PD-L1 agent and in over $1.0 \%$ of those treated with an anticytotoxic T-lymphocyte antigen-4 (CTLA-4) plus an anti-PD-1/anti-PD-L1 agent [9]. Much attention should be paid to immune-related adverse effects but without overweighing the benefits, that is, pathological complete response in this case.

With the remarkable clinical outcomes using various immune checkpoint inhibitors, the therapeutic strategy for inoperable NSCLC has diverged into two directions. When PD-L1 expression of the NSCLC is very high, that is, $\geq 50$ percent expression, dual immune checkpoint inhibitor therapy without chemotherapy plays an important role in order both to maintain quality of life for the patients and to possibly prolong survival of the patients. On the other

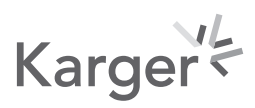


hand, in case of positive but weak PD-L1 expression, chemoimmunotherapy using platinum doublet and an immune checkpoint inhibitor, especially pembrolizumab, has become a standard therapeutic option for NSCLC not with curative intent but with palliative intent. However, the therapeutic goal of the locally advanced NSCLC should be changed from palliation to cure when the induction chemoimmunotherapy brings about a remarkable response.

In conclusion, physicians should take the induction therapy using platinum doublet and pembrolizumab into consideration to optimally treat the patients with advanced NSCLC, especially with low PD-L1 expression and paraneoplastic syndrome, followed by adequate local therapy. Further study with a pembrolizumab-containing chemoimmunotherapy should be done, with curative intent, in patients with locally advanced NSCLC.

\section{Statement of Ethics}

We have reported this case in compliance with the Declaration of Helsinki. Written informed consent was obtained from the patient for the publication of this case report and any accompanying images.

\section{Conflict of Interest Statement}

The authors have no conflicts of interest to declare.

\section{Funding Sources}

Not applicable.

\section{Author Contributions}

N.K. contributed the design of the report and collected the data. S.O. drafted the manuscript. T.Y. developed the therapeutic strategy of the patient. S.M. revised the manuscript. All authors read and approved the final version of the manuscript.

\section{References}

1 Hanna NH, Schneider BJ, Temin S, Baker JS, Brahmer J, Ellis PM, et al. Therapy for stage IV non-small-cell lung cancer without driver alterations: ASCO and OH (CCO) joint guideline update. J Clin Oncol. 2020;38:1608-32.

2 Ito T, Goto K, Yoh K, Niho S, Ohmatsu H, Kubota K, et al. Hypertrophic pulmonary osteoarthropathy as a paraneoplastic manifestation of lung cancer. J Thorac Oncol. 2010;5(7):976-80.

3 Barlesi F, Scherpereel A, Gorbunova V, Gervais R, Vikström A, Chouaid C, et al. Maintenance bevacizumabpemetrexed after first-line cisplatin-pemetrexed-bevacizumab for advanced nonsquamous nonsmall-cell lung cancer: updated survival analysis of the AVAPERL (M022089) randomized phase III trial. Ann Oncol. 2014; 25(5):1044-52.

4 Hellmann MD, Chaft JE, William WNJ, Rusch V, Pisters KMW, Kalhor N, et al. Pathologic response after neoadjuvant chemotherapy in resectable non-small cell lung cancers: proposal for the use of "major pathologic response" as a surrogate endpoint. Lancet Oncol. 2014;15:e42-e50.

5 Reck M, Rodriguez-Abreu D, Robinson AG, Hui R, Csoszi T, Fulop A, et al. Updated analysis of KEY-NOTE-024: Pembrolizumab versus platinum-based chemotherapy for advanced non-small-cell lung cancer with PD-L1 tumor proportion score of 50\% or greater. J Clin Oncol. 2019;37:537-46.

6 Paz-Ares L, Luft A, Vicente D, Tafreshi A, Gümüş M, Mazières J, et al. Pembrolizumab plus chemotherapy for squamous non-small-cell lung cancer. N Engl J Med. 2018;379(21):2040-51.

\section{Karger'}


7 Ilias I, Torpy DJ, Pacak K, Mullen N, Wesley RA, Nieman LK, et al. Cushing's syndrome due to ectopic corticotropin secretion: twenty years' experience at the national institutes of health. J Clin Endcrinol. 2005;90:4955-62.

8 Naidoo J, Page DB, Li BT, Connell LC, Schindler K, Lacouture M, et al. Toxicities of the anti-PD-1 and anti-PD-L1 immune checkpoint antibodies. Ann Oncol. 2015;26(12):2375-91.

9 Wang DY, Salem JE, Cohen JV, Chandra S, Menzer C, Ye F, et al. Fatal toxic effects associated with immune checkpoint inhibitors: a systematic review and meta-analysis. JAMA Oncol. 2018;4(12):1721-8. 